\title{
A Reliable Numerical Scheme for a Computer Virus Propagation Model
}

\author{
Pham Hoai Thu', Hoang Manh Tuan ${ }^{2}$ \\ ${ }^{1}$ Faculty of Information Security, People's Security Academy, Hanoi, Vietnam ${ }^{2}$ Department of Mathematics, FPT \\ University, Hoa Lac Hi-Tech Park, Km29 Thang Long Blvd, Hanoi, Vietnam \\ Correspondence: Hoang Manh Tuan, tuanhm14@fe.edu.vn; hmtuan01121990@gmail.com \\ Date received: 28/08/2021, Date revised: 29/10/2021, Date accepted: 15/11/2021 \\ Digital Object Identifier: 10.32913/mic-ict-research-vn.v2021.n2.1007
}

\begin{abstract}
In this paper, we develop the Mickens' methodology to construct a dynamically consistent Non-Standard Finite Difference (NSFD) scheme for a recognized computer virus propagation model. It is proved that the constructed NSFD scheme correctly preserves essential mathematical features of the continuous-time model, which are positivity, boundedness and asymptotic stability. As an important consequence, we obtain an effective numerical scheme that has the ability to provide reliable approximations, meanwhile, some typical standard finite difference schemes fail to preserve the essential properties of the computer virus propagation model; hence, they can generate numerical approximations which are not only negative but also unstable. Finally, a set of numerical experiments is performed to support the theoretical results as well as to demonstrate the advantage of the NSFD scheme over standard ones. As we expected, there is a good agreement between the numerical results and theoretical assertions.
\end{abstract}

Keywords: Computer virus, NSFD scheme, positivity, stability, dynamics consistency

Title: Một phương pháp hiệu quả mô phỏng số mô hình lan truyển virus máy tính

Abstract: Trong bài báo này, chúng tôi phát triển phương pháp luận của Mickens để xây dựng lược đồ sai phân phi tiêu chuẩn tương thích động lực với một mô hình lan truyền virus máy tính. Các phân tích toán học chỉ ra rằng lược đồ sai phân phi tiêu chuẩn bảo toàn các tính chất quan trọng của mô hình liên tục, bao gồm tính dương, tính bị chặn và tính chất ổn định tiệm cận. Trong khi đó, một số lược đồ sai phân tiêu chuẩn không thể bảo toàn các tính chất quan trọng của mô hình liên tục. Do đó, chúng có thể sinh ra các lời giải xấp xỉ âm và không ổn định. Các mô phỏng số được thực hiện để hỗ trợ và minh họa cho các kết quả lý thuyết cũng như để chứng minh ưu thế của lược đồ sai phân phi tiều chuẩn so với các lược đồ sai phân tiêu chuẩn. Các mô phỏng số chỉ ra rằng các kết quả số là tương thích với các kết quả lý thuyết.

Keywords: Computer virus, NSFD scheme, positivity, stability, dynamics consistency.

\section{INTRODUCTION}

The study of numerical methods for solving differential equations has played an important role not only in theory but also in practice. Over the past of several decades, a large number of numerical methods have been proposed and developed by mathematicians and engineers (see, for example, [1-3]). The concept of Non-Standard Finite Difference schemes was first introduced by Mickens to overcome drawbacks of Standard Finite Difference (SFD) schemes [4-8]. One of the main advantages of NSFD schemes is that they can preserve essential mathematical features of differential equations models (positivity, stability, monotonicity, periodicity, physical properties, etc.) regardless of chosen step sizes. Nowadays, NSFD schemes have been widely used as a powerful and effective approach to solve differential equation models that describe important phenomena and processes arising in science and technology. Good reviews of NSFD schemes can be found in [7, 9, 10]. Also, we refer the readers to [11-15] for some recent notable results on NSFD schemes for numerical approximations of differential equation models. Recently, we have successfully developed the Mickens' methodology to formulate NSFD schemes for mathematical models arising in real-world situations [16$22]$.

Nowadays, computer viruses have been one of the biggest threats to Internet security as well as to work and daily life. Therefore, effective strategies and measures for preventing and controlling computer viruses are always needed. For 
this purpose, many mathematical models, based on the high similarity between computer viruses and biological ones, have been constructed and developed to study the spread of computer viruses (see, for example, [23-28] and references therein). From the analysis of these models, we can propose effective strategies and measures for preventing and controlling computer viruses.

Motivated by the importance of computer virus propagation models and their numerical approximations, in this work we consider a recognized computer virus propagation model proposed by Gan et al. in a previous work [25]. The mathematical model and its dynamics will be provided in Section II. Although this computer virus propagation model has many useful applications in information technology and computer science and it has been studied and developed at some levels (see, for example, [23, 24]), to the best of our knowledge, dynamically consistent discrete-time models have not been constructed yet. Therefore, our objective is to construct a discrete-time model preserving essential qualitative features of the continuous-time model for all finite step sizes. For this purpose, the Mickens' methodology is applied to propose an appropriate NSFD scheme for the continuous-time model. After that, the positvity, boundedness and asymptotic stability of the proposed NSFD scheme are established. As a result, we obtain a dynamically consistent NSFD scheme that is able to provide reliable numerical approximations for the computer virus propagation model. In addition to the theoretical results, a set of numerical experiments is performed to support the theoretical results and to show advantages of the NSFD scheme over some typical SFD schemes. As will be seen in Section IV, the standard Euler and second-order Runge-Kutta (RK2) schemes fail to correctly preserve the dynamical properties of the continuous model for some given step sizes. More clearly, they generate numerical approximations that are not only negative but also unstable. Conversely, numerical approximations generated by the NSFD scheme faithfully reflect the dynamics of the continuous model regardless of chosen step sizes.

The plan of the paper is as follows:

The mathematical model of the computer virus propagation model is provided in Section II. The NSFD scheme is proposed and analyzed in Section III. Section IV reports some numerical experiments. Some conclusions and discussions are presented in the last section.

\section{MATHEMATICAL MODEL AND ITS DYNAM- ICS}

In this section, we briefly recall from [25] the mathematical model of the computer virus propagation model and its dynamics. In this model, at any time, a computer is called internal or external depending on it is accessed to the Internet or not. All internal computers are classified into two compartments:

1) $S$ compartment: The set of all uninfected internal computers or susceptible internal computers.

2) I compartment: The set of all infected internal computers.

Besides, all external computers are categorized into a single compartment:

1) $E$ compartment: The set of all external computers.

Let $S(t), I(t)$, and $E(t)$ be the the average numbers of susceptible, infected and external computers at time $t$, respectively. Based on some reasonable hypotheses (see the hypotheses $\left(A_{1}\right)-\left(A_{7}\right)$ in [25]), the following differential equation model was proposed:

$$
\begin{aligned}
& \dot{S}=\gamma_{2} I+\eta_{2} E-\mu S-\beta S I-\gamma_{1} S, \\
& \dot{I}=\beta S I-\mu I-\gamma_{1} I-\gamma_{2} I+\eta_{1} E, \\
& \dot{E}=\delta+\gamma_{1} S+\gamma_{1} I-\mu E-\eta_{1} E-\eta_{2} E,
\end{aligned}
$$

where all the parameters are assumed to be positive. We refer the readers to [25] for more details of this model. It was proved in [25] that:

1) The model (1) has no virus-free equilibrium point.

2) The model (1) possesses a unique viral equilibrium point $E_{V}^{*}=\left(S^{*}, I^{*}, E^{*}\right)$, where

$$
\begin{aligned}
E^{*} & =\frac{\delta\left(\mu+\gamma_{1}\right)}{\mu\left(\mu+\gamma_{1}+\eta_{1}+\eta_{2}\right)}, \\
I^{*} & =\frac{\beta w-\left(\mu+\gamma_{1}+\gamma_{2}\right)}{2 \beta} \\
& +\frac{\sqrt{\left[\beta w-\left(\mu+\gamma_{1}+\gamma_{2}\right)\right]^{2}+4 \beta \eta_{1} E^{*}}}{2 \beta}, \\
S^{*} & =\frac{\delta}{\mu}-E^{*}-I^{*},
\end{aligned}
$$

with

$$
w:=\frac{\delta\left(\gamma_{1}+\gamma_{2}\right)}{\mu\left(\mu+\gamma_{1}+\eta_{1}+\eta_{2}\right)} .
$$

3) The viral equilibrium point $E_{V}^{*}$ is not only locally asymptotically stable but globally asymptotically stable with respect to the positively invariant set

$$
\Omega:=\left\{(S, I, E) \mid S, I, E \geq 0, S+I+E \leq \frac{\delta}{\mu}\right\} .
$$

\section{THE CONSTRUCTION OF NSFD SCHEME}

Consider the continuous-time model (1) on a finite interval $[0, T]$ and discretize this interval by a uniform mesh

$$
0=t_{0}<t_{1}<\ldots<t_{i}<\ldots<t_{M-1}<t_{M}=T,
$$


where $t_{n+1}-t_{n}=\Delta t$ for $i=0,1, \ldots, M-1$. Let $\left(S_{n}, I_{n}, R_{n}\right) \quad(n=1,2, \ldots, M)$ be the intended approximations for $\left(S\left(t_{n}\right), I\left(t_{n}\right), R\left(t_{n}\right)\right)$, respectively. Using the Mickens' methodology [4-8], we discretize the model (1) as follows

$$
\begin{aligned}
\dot{S}\left(t_{n}\right) & \approx \frac{S_{n+1}-S_{n}}{\varphi(\Delta t)}, \\
\dot{I}\left(t_{n}\right) & \approx \frac{I_{n+1}-I_{n}}{\varphi(\Delta t)}, \\
\dot{E}\left(t_{n}\right) & \approx \frac{E_{n+1}-E_{n}}{\varphi(\Delta t)},
\end{aligned}
$$

and

$$
\begin{aligned}
& \gamma_{2} I\left(t_{n}\right)+\eta_{2} E\left(t_{n}\right)-\mu S\left(t_{n}\right)-\beta S\left(t_{n}\right) I\left(t_{n}\right)-\gamma_{1} S\left(t_{n}\right) \\
& \approx \gamma_{2} I_{n}+\eta_{2} E_{n}-\mu S_{n+1}-\beta S_{n+1} I_{n}-\gamma_{1} S_{n+1}, \\
& \beta S\left(t_{n}\right) I\left(t_{n}\right)-\mu I\left(t_{n}\right)-\gamma_{1} I\left(t_{n}\right)-\gamma_{2} I\left(t_{n}\right)+\eta_{1} E\left(t_{n}\right) \\
& \approx \beta S_{n+1} I_{n}-\mu I_{n+1}-\gamma_{1} I_{n+1}-\gamma_{2} I_{n}+\eta_{1} E_{n}, \\
& \delta+\gamma_{1} S\left(t_{n}\right)+\gamma_{1} I\left(t_{n}\right)-\mu E\left(t_{n}\right)-\eta_{1} E\left(t_{n}\right)-\eta_{2} E\left(t_{n}\right) \\
& \approx \delta+\gamma_{1} S_{n+1}+\gamma_{1} I_{n+1}-\mu E_{n+1}-\eta_{1} E_{n}-\eta_{2} E_{n},
\end{aligned}
$$

where $\varphi(\Delta t)$ is a positive function satisfying $\varphi(\Delta t)=\Delta t+$ $O\left(\Delta t^{2}\right)$ as $\Delta t \rightarrow 0$. For convenience, the variable $\Delta t$ will be omitted in some presentations. The discretization process (3)-(4) leads to the following NSFD scheme for the model (1)

$$
\begin{aligned}
\frac{S_{n+1}-S_{n}}{\varphi(\Delta t)} & =\gamma_{2} I_{n}+\eta_{2} E_{n}-\mu S_{n+1} \\
& -\beta S_{n+1} I_{n}-\gamma_{1} S_{n+1}, \\
\frac{I_{n+1}-I_{n}}{\varphi(\Delta t)} & =\beta S_{n+1} I_{n}-\mu I_{n+1}-\gamma_{1} I_{n+1} \\
& -\gamma_{2} I_{n}+\eta_{1} E_{n}, \\
\frac{E_{n+1}-E_{n}}{\varphi(\Delta t)} & =\delta+\gamma_{1} S_{n+1}+\gamma_{1} I_{n+1}-\mu E_{n+1} \\
& -\eta_{1} E_{n+1}-\eta_{2} E_{n} .
\end{aligned}
$$

We now investigate dynamical properties of the NSFD model (5). Let us define a sequence $\left\{N_{n}\right\}_{n=0}^{\infty}$ with

$$
N_{n}:=S_{n}+I_{n}+R_{n}, \quad n \geq 0 .
$$

Theorem 1 (Positivity and boundedness): Let $\varphi(\Delta t)$ be a function satisfying

$$
\varphi(\Delta t)<\Delta t^{*}:=\min \left\{\frac{1}{\gamma_{2}}, \frac{1}{\eta_{1}+\eta_{2}}\right\} \text { for all } h>0 .
$$

Then, we have the following assertions:

1) The set $\mathbb{R}_{3}^{+}=\{(S, I, R) \mid S, I, E \geq 0\}$ is a positively invariant set of the NSFD scheme (5), i.e., $S_{n+1}, I_{n+1}, E_{n+1} \geq 0$ whenever $S_{n}, I_{n}, E_{n} \geq 0$.

2) The sequence $\left\{N_{n}\right\}_{n=0}^{\infty}$ monotonically converges to $N^{*}:=\delta / \mu$ as $n \rightarrow \infty$.
Proof: Proof of Part 1. First, we rewrite the system (5) in the explicit form:

$$
\begin{aligned}
S_{n+1} & =\frac{S_{n}+\varphi \gamma_{2} I_{n}+\varphi \eta_{2} E_{n}}{1+\varphi\left(\mu+\beta I_{n}+\gamma_{1}\right)} \\
I_{n+1} & =\frac{\left(1-\varphi \gamma_{2}\right) I_{n}+\varphi \beta S_{n+1} I_{n}+\varphi \eta_{1} E_{n}}{1+\varphi\left(\mu+\gamma_{1}\right)}, \\
E_{n+1} & =\frac{\varphi \delta+\varphi \gamma_{1}\left(S_{n+1}+I_{n+1}\right)+\left[1-\varphi\left(\eta_{1}+\eta_{2}\right)\right] E_{n}}{1+\varphi \mu}
\end{aligned}
$$

The condition (6) implies that $1-\varphi \gamma_{2}>0$ and $1-\varphi\left(\eta_{1}+\right.$ $\left.\eta_{2}\right)>0$. So, we deduce that $S_{n+1}, I_{n+1}, E_{n+1} \geq 0$ whenever $S_{n}, I_{n}, E_{n} \geq 0$. The proof of this part is complete.

Proof of Part 2. Adding side-by-side the 1st, 2nd and 3rd equations of the system (5) we obtain

$$
\frac{N_{n+1}-N_{n}}{\varphi}=\delta-\mu N_{n+1}
$$

which implies that

$$
N_{n+1}=\left(\frac{\delta}{\mu}-N_{0}\right)\left(\frac{1}{1+\varphi \mu}\right)^{n}+\frac{\delta}{\mu} .
$$

Consequently, the monotone convergence of the sequence $\left\{N_{n}\right\}$ is proved by using the fact that $1 /(1+\varphi \mu) \in(0,1)$. The proof of this part is completed.

Next, to determine the set of equilibria of the model (5), we need to solve the following system

$$
S_{n+1}=S_{n}, \quad I_{n+1}=I_{n}, \quad E_{n+1}=E_{n} .
$$

It is easy to verify that equilibrium points of the models (5) and (1) are identical. Hence, the NSFD model (5) only possesses a unique viral (positive) equilibrium point $E_{V}^{*}$, which is given by (2).

Theorem 2 (Asymptotic stability): Let $\varphi(\Delta t)$ be a function satisfying the condition (6). Then, the viral equilibrium point $E_{V}^{*}$ is a locally asymptotically stable equilibrium point of the NSFD model (5).

Proof: First, using the relation $S_{n}=N_{n}-I_{n}-E_{n}$ and the equation (8) we transform the system (5) to the form

$$
\begin{aligned}
I_{n+1} & =\frac{\left(1-\varphi \gamma_{2}\right) I_{n}+\varphi \beta\left(N_{n+1}-I_{n+1}-E_{n+1}\right) I_{n}+\varphi \eta_{1} E_{n}}{1+\varphi\left(\mu+\gamma_{1}\right)} \\
E_{n+1} & =\frac{\varphi \delta+\varphi \gamma_{1}\left(N_{n+1}-E_{n+1}\right)+\left[1-\varphi\left(\eta_{1}+\eta_{2}\right)\right] E_{n}}{1+\varphi \mu} \\
N_{n+1} & =N_{n}+\varphi \delta-\varphi \mu N_{n+1} .
\end{aligned}
$$

Then, the viral equilibrium point $E_{V}^{*}=\left(S^{*}, I^{*}, E^{*}\right)$ of (5) is transformed to $E_{\text {new }}^{*}=\left(I^{*}, E^{*}, \delta / \mu\right)$. We need to show that 
$E_{\text {new }}^{*}$ is a locally asymptotically stable equilibrium point of the model (9). Indeed, let us denote

$$
\begin{aligned}
& \lambda_{1}:=\frac{1-\varphi \gamma_{2}+\varphi \beta S^{*}}{\left[1+\frac{\varphi \beta I^{*}}{1+\varphi\left(\mu+\gamma_{1}\right)}\right]\left[1+\varphi\left(\mu+\gamma_{1}\right)\right]}, \\
& \lambda_{2}:=\frac{1-\varphi\left(\eta_{1}+\eta_{2}\right)}{1+\varphi\left(\mu+\gamma_{1}\right)}, \\
& \lambda_{3}:=\frac{1}{1+\varphi \mu} .
\end{aligned}
$$

Then, the Jacobian matrix of the system (9) at $E_{\text {new }}^{*}$ is given by

$$
J\left(E_{\text {new }}^{*}\right)=\left(\begin{array}{ccc}
\lambda_{1} & \frac{\partial I_{n+1}}{\partial E_{n}}\left(E_{\text {new }}^{*}\right) & \frac{\partial I_{n+1}}{\partial N_{n}}\left(E_{\text {new }}^{*}\right) \\
0 & \lambda_{2} & \frac{\partial E_{n+1}}{\partial N_{n}}\left(E_{\text {new }}^{*}\right) \\
0 & 0 & \lambda_{3}
\end{array}\right) .
$$

Consequently, $\lambda_{1}, \lambda_{2}$ and $\lambda_{3}$ are all the eigenvalues of $J\left(E^{*}\right)$. It is clear that $\left|\lambda_{3}\right|<1$. On the other hand, the condition (6) implies that $\left|\lambda_{2}\right|<1$. We now show that $\left|\lambda_{1}\right|<1$. Indeed, it follows from (6) that $\lambda_{1}>0$. Moreover, from the second equation of the system (1) we obtain

$1-\lambda_{1}=\varphi \frac{\mu+\gamma_{1}+\gamma_{2}+\beta I^{*}-\beta S^{*}}{1+\varphi\left(\mu+\gamma_{1}\right)+\varphi \beta I^{*}}=\varphi \frac{\beta I^{*}+\eta_{1} E^{*} / I^{*}}{1+\varphi\left(\mu+\gamma_{1}\right)+\varphi \beta I^{*}}$, which means that $\lambda_{1}<0$. Therefore, $0<\lambda_{1}<1$.

So, all the eigenvalues of $J\left(E_{n e w}^{*}\right)$ are inside the unit ball. By the Lyapunov indirect method [29, 30] we obtain the local asymptotic stability of $E_{n e w}^{*}$. The proof is completed.

\section{Remark 1:}

1) Theorems 1 and 2 mean that the NSFD scheme (5) preserves the positvity, boundedness and asymptotic stability of the model (1) for all finite step sizes $\Delta t>$ 0 .

2) There are many denominator functions $\varphi(\Delta t)$ satisfying the condition (6), a typical function is (see [4-8])

$$
\varphi(\Delta t)=\frac{1-e^{-\tau \Delta t}}{\tau}, \quad \tau>\frac{1}{\Delta t^{*}} .
$$

General families of denominator functions satisfying (6) can be found in [18].

3) Numerical examples in Section IV show that the NSFD scheme (5) preserves not only local asymptotic stability but also global asymptotic stability of the model (1).

\section{NUMERICAL EXPERIMENTS}

In this section, we reports some numerical examples to support the theoretical results as well as to show the advantage of the NSFD scheme over standard ones. For this purpose, we consider the model (1) with the following data.

Table I

PARAMETERS USED IN NUMERICAL EXAMPLES

\begin{tabular}{cccccccc}
\hline Case & $\beta$ & $\delta$ & $\mu$ & $\eta_{1}$ & $\eta_{2}$ & $\gamma_{1}$ & $\gamma_{2}$ \\
\hline 1 & 0.05 & 0.8 & 0.01 & 0.75 & 0.85 & 0.6 & 0.8 \\
2 & 0.05 & 0.02 & 0.01 & 0.8 & 0.9 & 0.6 & 0.8 \\
\hline
\end{tabular}

Example 1: Consider the model (1) with the parameters given by Case 1 in Table I. In this case, the viral equilibrium point $E_{V}^{*}=(19.56,38.36,22.08)$ is asymptotically stable. Numerical approximations generated by the standard Euler scheme, RK2 scheme and NSFD scheme are depicted in Figures 1-4, respectively. It is clear that the Euler and RK2 schemes fail to preserve the asymptotic stability of the continuous model, meanwhile, the NSFD scheme correctly preserves the dynamics of the continuous model. Furthermore, the dynamics of NSFD scheme does not depend on the chosen step sizes.

Example 2: Consider the model (1) with the parameters given by Case 2 in Table I. In this case, the viral equilibrium point $E_{V}^{*}=(1.16,0.31,0.52)$ is asymptotically stable. As shown in Figure 5, the Euler and RK2 schemes destroy not only the positivity but also asymptotic stability, meanwhile, the NSFD scheme preserve all the mathematical features of the continuous model.

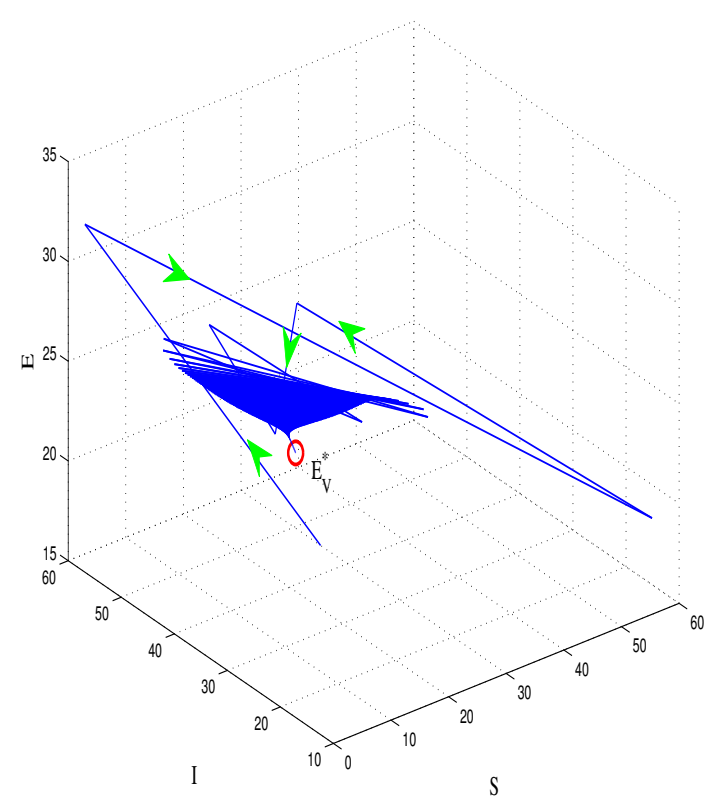

Figure 1. The phase space generated by the Euler scheme for $(S(0), I(0), E(0))=(30,45,15)$ and $\Delta t=0.8$ after 1000 iterations. 


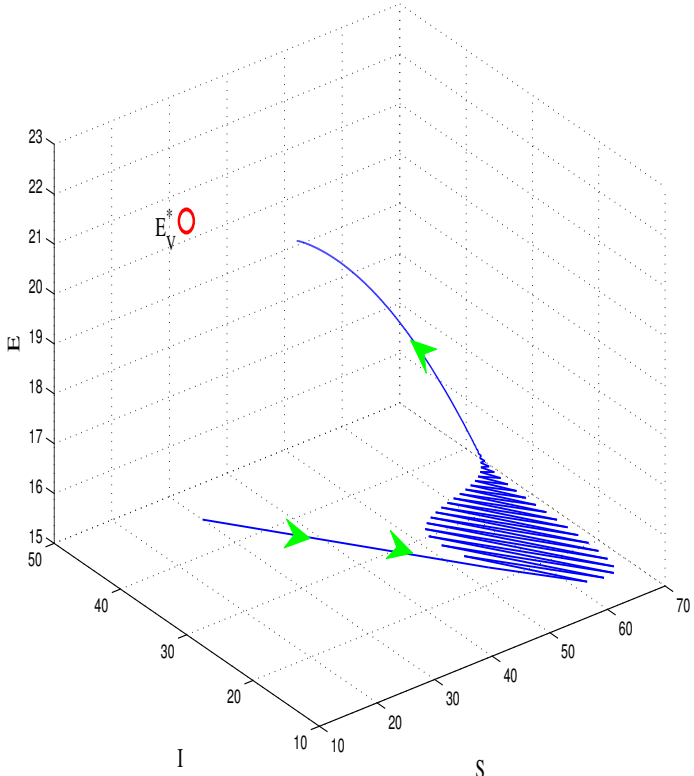

Figure 2. The phase space generated by the RK2 scheme for $(S(0), I(0), E(0))=(30,45,15)$ and $\Delta t=0.9$ after 1000 iterations.

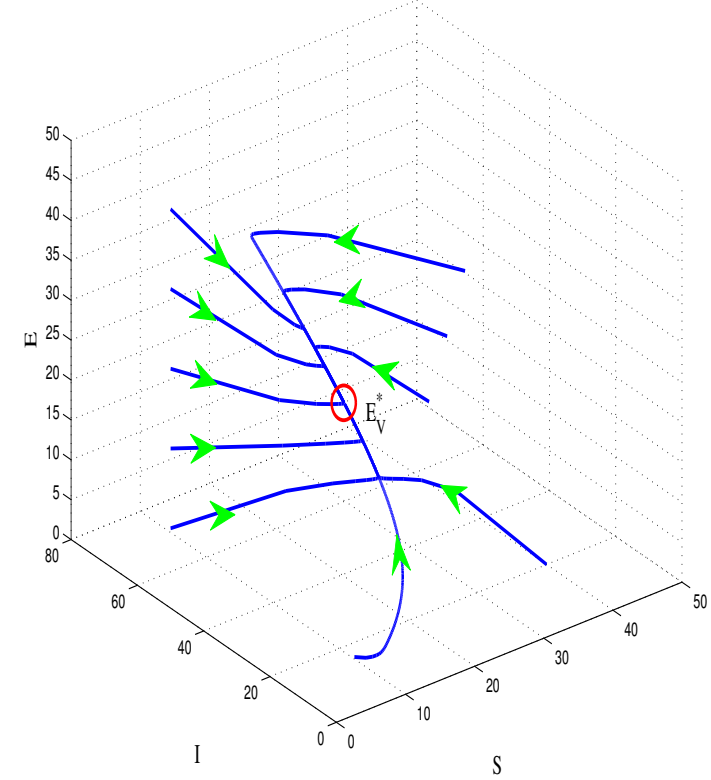

Figure 3. The phase spaces generated by the NSFD scheme for $\Delta t=1$ and $\varphi(\Delta t)=\frac{1-e^{-1.6 \Delta t}}{1.6}$ after 1000 iterations. Each blue curve represents a phase space corresponding to a specific initial data, the red circle represents the viral equilibrium point and the green arrows show the evolution of the model.

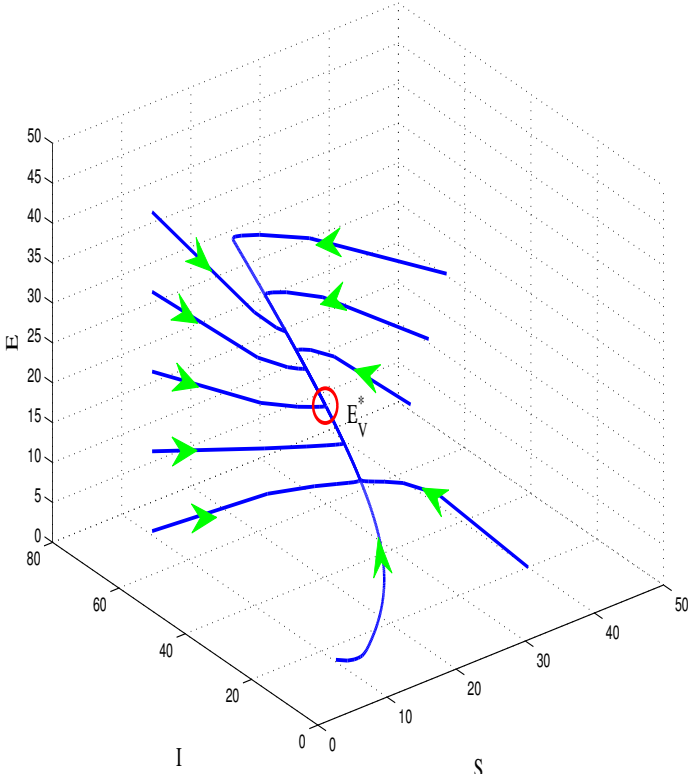

Figure 4 . The phase spaces generated by the NSFD scheme for $\Delta t=2$ and $\varphi(\Delta t)=\frac{1-e^{-1.6 \Delta t}}{1.6}$ after 1000 iterations.

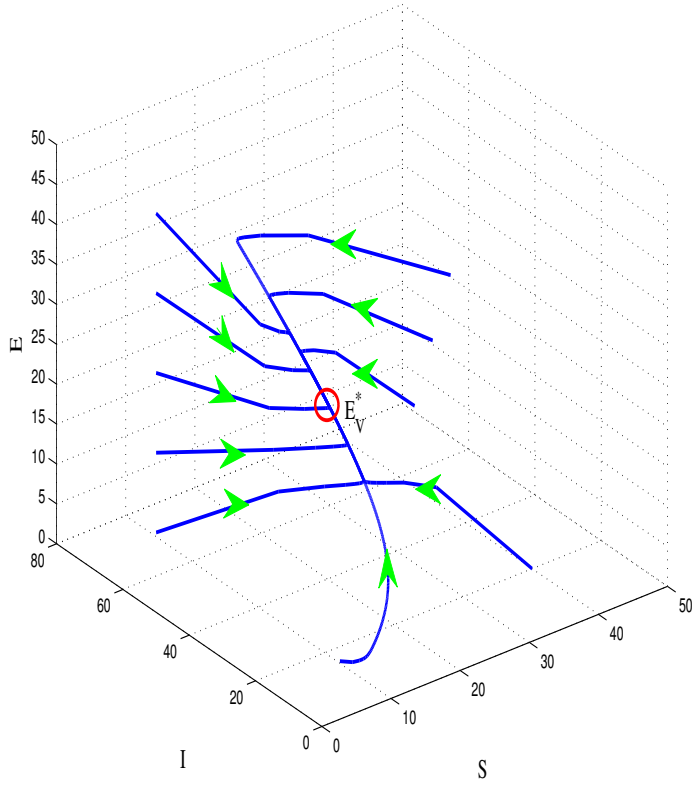

Figure 5. The phase spaces generated by the Euler scheme with $h=0.87$ after 100 iterations, the RK2 scheme with $h=0.88$ after 100 iterations and the NSFD scheme with $\Delta t=1$ and $\varphi(\Delta t)=$ $\frac{1-e^{-1.8 \Delta t}}{1.8}$ after 100 iterations.

From Examples 1 and 2, we see that the standard Euler 
and RK2 scheme cannot preserve the posivitiy and stability of the continuous model for all finite step sizes. A similar comment for the classical Runge-Kutta schemes can be found in some of our previous works [16-22]. Consequently, the advantage of the NSFD scheme over the standard Euler and RK2 schemes is that they can correctly preserve the dynamics of the continuous model regardless of the chosen step sizes. Hence, the NSFD scheme is appropriate and effective to simulate dynamics of the differential equation model over long time periods.

\section{CONCLUSIONS AND DISCUSSIONS}

In this paper, we have successfully developed the Mickens' methodology to construct a reliable NSFD scheme for the computer virus propagation model (1). The theoretical assertions have been supported by a set of numerical examples. It was proved that the constructed NSFD scheme preserves the positivity, boundedness and asymptotic stability of the continuous model for all finite step sizes. As a result, it provided reliable approximations, meanwhile, some typical standard schemes failed to preserve the essential mathematical features of the continuous model; consequently, they generated numerical solutions which are not only unstable but also negative.

In the near future, we will extend the approach and obtained results in this paper to construct dynamically consistent NSFD schemes for ordinary and fractional-order differential equation models arising in science and technology. In addition, the construction of high-order NSFD schemes for the computer virus propagation models will be considered.

\section{REFERENCES}

[1] U. M. Ascher and L. R. Petzold, Computer methods for ordinary differential equations and differential-algebraic equations. Siam, 1998, vol. 61.

[2] G. Wanner and E. Hairer, Solving ordinary differential equations II. Springer Berlin Heidelberg, 1996, vol. 375.

[3] G. Wanner, E. Hairer, and S. P. Norsett, Solving ordinary differential equations I. Springer Berlin Heidelberg, 1990.

[4] R. E. Mickens, Nonstandard Finite Difference Schemes: Methodology and Applications. World Scientific, 2020.

[5] _ - Advances in the applications of nonstandard finite difference schemes. World Scientific, 2005.

[6] _ , "Nonstandard finite difference schemes for differential equations," Journal of Difference Equations and Applications, vol. 8, no. 9, pp. 823-847, 2002.

[7] - Applications of nonstandard finite difference schemes. World Scientific, 2000

[8] — Nonstandard finite difference models of differential equations. world scientific, 1994

[9] K. Patidar, "Nonstandard finite difference methods: recent trends and further developments," Journal of Difference Equations and Applications, vol. 22, no. 6, pp. 817-849, 2016.
[10] K. C. Patidar, "On the use of nonstandard finite difference methods," Journal of Difference Equations and Applications, vol. 11, no. 8, pp. 735-758, 2005.

[11] O. Adekanye and T. Washington, "Nonstandard finite difference scheme for a tacoma narrows bridge model," Applied Mathematical Modelling, vol. 62, pp. 223-236, 2018.

[12] A. Korpusik, "A nonstandard finite difference scheme for a basic model of cellular immune response to viral infection," Communications in Nonlinear Science and Numerical Simulation, vol. 43, pp. 369-384, 2017.

[13] J. Martín-Vaquero, A. Martín del Rey, A. Encinas, J. Hernández Guillén, A. Queiruga-Dios, and G. Rodríguez Sánchez, "Higher-order nonstandard finite difference schemes for a mseir model for a malware propagation," Journal of Computational and Applied Mathematics, vol. 317, pp. 146-156, 2017.

[14] Y. Yang, J. Zhou, X. Ma, and T. Zhang, "Nonstandard finite difference scheme for a diffusive within-host virus dynamics model with both virus-to-cell and cell-to-cell transmissions," Computers and Mathematics with Applications, vol. 72, no. 4, pp. 1013-1020, 2016

[15] J. Cresson and F. Pierret, "Non standard finite difference scheme preserving dynamical properties," Journal of Computational and Applied Mathematics, vol. 303, pp. 15-30, 2016.

[16] M. T. Hoang, "Reliable approximations for a hepatitis b virus model by nonstandard numerical schemes," Mathematics and Computers in Simulation, vol. 193, pp. 32-56, 2022.

[17] Q. A. Dang and M. T. Hoang, "Positivity and global stability preserving nsfd schemes for a mixing propagation model of computer viruses," Journal of Computational and Applied Mathematics, vol. 374, p. 112753, 2020.

[18] — - "Positive and elementary stable explicit nonstandard runge-kutta methods for a class of autonomous dynamical systems," International Journal of Computer Mathematics, vol. 97, no. 10, pp. 2036-2054, 2020.

[19] — "Nonstandard finite difference schemes for a general predator-prey system," Journal of Computational Science, vol. 36, p. 101015, 2019.

[20] _ - "Complete global stability of a metapopulation model and its dynamically consistent discrete models," Qualitative theory of dynamical systems, vol. 18, no. 2, pp. 461-475, 2019.

[21] M. T. Hoang and A. Nagy, "Uniform asymptotic stability of a logistic model with feedback control of fractional order and nonstandard finite difference schemes," Chaos, Solitons \& Fractals, vol. 123, pp. 24-34, 2019.

[22] Q. A. Dang and M. T. Hoang, "Lyapunov direct method for investigating stability of nonstandard finite difference schemes for metapopulation models," Journal of Difference Equations and Applications, vol. 24, no. 1, pp. 15-47, 2018.

[23] C. Gan, X. Yang, and Q. Zhu, "Propagation of computer virus under the influences of infected external computers and removable storage media," Nonlinear Dynamics, vol. 78, no. 2, pp. 1349-1356, 2014.

[24] C. Gan, X. Yang, W. Liu, Q. Zhu, J. Jin, and L. He, "Propagation of computer virus both across the internet and external computers: a complex-network approach," Communications in Nonlinear Science and Numerical Simulation, vol. 19, no. 8, pp. 2785-2792, 2014.

[25] C. Gan, X. Yang, Q. Zhu, J. Jin, and L. He, "The spread of computer virus under the effect of external computers," Nonlinear Dynamics, vol. 73, no. 3, pp. 1615-1620, 2013.

[26] C. Gan, X. Yang, W. Liu, Q. Zhu, and X. Zhang, "An epidemic model of computer viruses with vaccination and generalized nonlinear incidence rate," Applied Mathematics and Computation, vol. 222, pp. 265-274, 2013. 
[27] J. Ren, X. Yang, Q. Zhu, L.-X. Yang, and C. Zhang, "A novel computer virus model and its dynamics," Nonlinear Analysis: Real World Applications, vol. 13, no. 1, pp. 376384, 2012.

[28] J. R. C. Piqueira and V. O. Araujo, "A modified epidemiological model for computer viruses," Applied Mathematics and Computation, vol. 213, no. 2, pp. 355-360, 2009.

[29] S. Elaydi, An Introduction to Difference Equations. Springer, 2005

[30] H. K. Khalil, Nonlinear systems third edition, 2002, vol. 115.

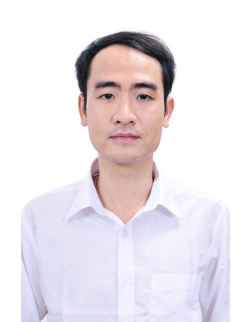

Hoang Manh Tuan received the Ph.D. degree in Applied Mathematics from Graduate University of Science and Technology, Vietnam Academy of Science and Technology (VAST) in 2021, the M.S in Applied Mathematics in 2015 and the B.S degree in Mathematics in 2012 from VNU University of Science. Currently, he is a lecturerresearcher at the Department of Mathematics, FPT University. His research interests are the qualitative theory and numerical analysis of differential equations and mathematical methods in information technology.

Email: tuanhm@fpt.edu.vn; hmtuan01121990@gmail.com

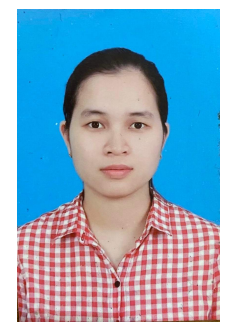

Pham Hoai Thu received the M.S in Information Systems from Posts and Telecommunications Institute of Technology in 2019 and the B.S in Informatics from People's Security Academy in 2014. Currently, she is a lecturer at the Faculty of Information Security, People's Security Academy. Her research interests include mathematical foundations for computer science and database.

Email: phamthuhvan@gmail.com 